EDNa JoAna CláUdio ManRIQUE ${ }^{1}$

Suelene Brito do Nascimento Tavares ${ }^{2}$

Nadja Lindany Alves SOUZA²

Zair Benedita Pinheiro Albuquerque ${ }^{2}$

LuIz Carlos ZEFerinO ${ }^{3}$

Rita GORETI AMARAL ${ }^{4}$

Artigos originais

Palavras-chaves

Neoplasias do colo do útero/

diagnóstico

Citodiagnóstico

Reações falso-negativas

Programas de rastreamento

Controle de qualidade

Garantia da qualidade dos cuidados de saúde/métodos

Keywords

Uterine cervical neoplasms/diagnosis Cytodiagnosis

False negative reactions

Mass screening

Quality control

Quality assurance, health care/ methods

\section{A revisão rápida de 100\% é eficiente na detecção de resultados falsos-negativos dos exames citopatológicos cervicais e varia com a adequabilidade da amostra: uma experiência no Brasil}

\author{
The 100\% rapid rescreening is effcicient in the detection \\ of false-negative results and varies according to the quality \\ of the sample: a Brazilian experience
}

\section{Resumo}

OBJETIVO: avaliar a eficiência da revisão rápida de 100\% para detecção de resultados falsos-negativos dos exames citopatológicos cervicais e verificar se esses resultados variam com a adequabilidade da amostra e com a idade da mulher. MÉTODOS: para avaliar a eficiência da revisão rápida, os 5.530 esfregaços classificados como negativos pelo escrutínio de rotina, após serem submetidos à revisão rápida de 100\%, foram comparados com as revisões dos esfregaços com base em critérios clínicos e aleatória de 10\%. Para análise estatística, as variáveis foram estudadas de maneira descritiva e, quando houve comparação, foram aplicados o teste do $x^{2}$ e o teste Cochran-Armitage. RESULTADOS: dos 141 esfregaços suspeitos pela revisão rápida, 84 (59,6\%) foram confirmados pelo diagnóstico final; desses, 36 $(25,5 \%)$ foram classificados como células escamosas atípicas de significado indeterminado, cinco $(3,5 \%)$ como células escamosas atípicas, não podendo excluir lesão de alto grau, 34 (24, 1\%) como lesão intra-epitelial escamosa de baixo grau, seis $(4,3 \%)$ como lesão intra-epitelial de alto grau e três $(2,1 \%)$ como células glandulares atípicas. Dos 84 esfregaços suspeitos e confirmados pelo diagnóstico final, 62 (73,8) foram classificados como satisfatórios e $22(26,2 \%)$ satisfatórios, porém com alguma limiłação, mas não se observou diferença significativa com a idade da mulher. CONCLUSÕES: os resultados deste estudo mostraram que a revisão rápida é uma alternativa eficiente como método de controle interno da qualidade na detecção de resultados falsos-negativos dos exames citopatológicos cervicais. Observou-se, também, que a revisão rápida apresentou melhor desempenho quando a amostra foi classificada como satisfatória para análise, porém não variou com a idade da mulher.

\section{Abstract}

PURPOSE: to evaluate the efficiency of the 100\% rapid rescreening in the detection of false-negative results and to verify whether the results vary according to the adequacy of the sample and the woman's age group. METHODS: to evaluate the efficiency of the rapid rescreening, the 5,530 smears classified as negative by the routine screening, after being submitted to the rapid rescreening of $100 \%$, were compared with the rescreening of the smears on the basis of clinical criteria and $10 \%$ random rescreening. For statistical analysis, the variables were evaluated descriptively and the $x^{2}$ test and the Cochran-Armitage test were applied to compare results. RESULTS: of the 141 smears identified as suspicious according to the rapid rescreening method, $84(59.6 \%)$ cases were confirmed in the final diagnosis, of which 36 (25.5\%) were classified as atypical squamous cells of undetermined significance, five (3.5\%) as atypical squamous cells that cannot exclude high-grade squamous intraepithelial lesion, 34 (24.1\%) as low-grade squamous intraepithelial lesion, six (4.3\%) as high-grade squamous intraepithelial lesion, and three $(2.1 \%)$ as atypical glandular cells. Of the 84 suspect smears confirmed in the final diagnosis, $62(73.8 \%)$ smears were classified as adequate and $22(26.2 \%)$ as adequate but with some limitation, but no significant difference was observed with the woman's age. CONCLUSIONS: the results of this study show that rapid rescreening is an efficient option for internal quality control for the detection of false-negative cervical smear results. In addition, it should be noted that rapid rescreening performed better when the sample was classified as adequate for analysis; however, it did not vary according to the woman's age group.
Correspondência:

Rita Goreti Amaral Avenida Belo Horizonte, Qd 39, Lt 04, Setor Joo CEP 74673-020 - Goiânia/G0 Fone: (62) 3209-6044 - Fax: (62) 3209-6037 E-mail: amaral@farmacia.ufg.br

Recebido 28/02/07
Laboratório de Análises Clínicas Rômulo Rocha da Faculdade de Farmácia da Universidade Federal de Goiás - UFG - Goiânia (GO), Brasil.

Apoio Financeiro: Conselho Nacional de Desenvolvimento Científico e Tecnológico (CNPq) 403402/2004-2.

Pós-Graduanda em Ciências da Saúde pela Universidade Federal de Goiás - UFG - Goiânia (GO), Brasil.

${ }_{2}^{2}$ Citologista da Faculdade de Farmácia da Universidade Federal de Goiás - UFG - Goiânia (GO), Brasil.

${ }^{3}$ Professor do Departamento de Tocoginecologia da Faculdade de Ciências Médicas da Universidade Estadual de Campinas -

UNICAMP - Campinas (SP), Brasil.

${ }_{4}^{4}$ Professora da Faculdade de Farmácia da Universidade Federal de Goiás - UFG - Goiânia (GO), Brasil.

Aceito com modificacậes 


\section{Introdução}

O exame citopatológico é o método mais utilizado nos programas de prevenção e detecção precoce do câncer do colo do útero. Entretanto, é necessário ter infra-estrutura complexa e muito bem organizada para obter resultados satisfatórios: unidades de saúde e profissionais bem treinados para coleta e preparo dos esfregaços de forma adequada, laboratório com profissionais habilitados para a realização dos exames, bem como profissionais responsáveis pelo seguimento das mulheres com resultados alterados ${ }^{1}$.

Apesar dos bons resultados do controle do câncer do colo do útero em muitos países desenvolvidos, esse tipo de câncer mantém-se como doença de alta prevalência, incidência e mortalidade em países onde os programas ainda não estão bem organizados ${ }^{2,3}$. O programa de controle de câncer do colo do útero pode falhar em vários pontos de sua estruturação, desde a coleta de material cervical até a perda de seguimento dos casos positivos ${ }^{3}$. As altas taxas de resultados falsosnegativos dos exames citopalógicos é um dos pontos de fragilidade que tem levado ao desenvolvimento de várias estratégias corretivas como a implementação de controle interno da qualidade ${ }^{4,5}$.

Os problemas provenientes de resultados falsosnegativos são relevantes. Para a paciente implica falsa segurança e atraso do diagnóstico, o que pode causar complicações sérias à saúde. Para o sistema de saúde, há gastos sem resultados ${ }^{1}$.

Os fatores que comprometem a adequabilidade da amostra também podem aumentar as taxas de resultados falsos-negativos, tais como a não representação de células endocervicais e/ou zona de transformação, a presença de sangue, processos inflamatórios e artefatos de fixação. Esses fatores normalmente retratam erros da coleta, que podem também causar erros de escrutínio e de interpretação ${ }^{6}$.

A revisão dos esfregaços interpretados previamente como negativos tem sido utilizada como controle interno da qualidade ${ }^{7-9}$. Nos Estados Unidos, o Clinical Laboratory Improvement Amendments of 1988 (Clia) recomenda a revisão aleatória de $10 \%$ dos esfregaços negativos. No entanto, esse método parece não ser eficaz para detectar as lesões não diagnosticadas no escrutínio de rotina ${ }^{10,11}$.

No Brasil, de acordo com as recomendações do Ministério da Saúde, os laboratórios deverão revisar pelo menos $10 \%$ dos exames realizados, os quais serão selecionados de acordo com o roteiro de critérios de risco clínico (hemorragia genital pós-menopausa, sangramento ectocervical de contato, evidência de doenças sexualmente transmissíveis ao exame ginecológico, alterações macroscópicas significativas ao exame especular ou à colposcopia, rádio ou quimioterapia prévias) e critérios citopatológicos (esfregaços com atipias escamosas ou glandulares e insatisfatórios em decorrência de hemorragia). Também devem ser incluídos na revisão pelo menos $5 \%$ de esfregaços negativos selecionados aleatoriamente ${ }^{4}$.

$\mathrm{Na}$ Europa, especialmente no Reino Unido, a revisão rápida de $100 \%$ foi introduzida desde a década de 1990 como método de controle interno da qualidade. Esse método consiste em revisar rapidamente todos os esfregaços classificados previamente como negativos no escrutínio de rotina. Após a revisão rápida, os esfregaços identificados como suspeitos são revisados detalhadamente por um profissional experiente que definirá o resultado final ${ }^{11-13}$.

Vários estudos mostraram que esse método apresenta bom desempenho na detecção de resultados falsos-negativos ${ }^{7-9,11,14-16}$. Todavia, falta informação se o desempenho do método de revisão rápida de $100 \%$ varia em função da adequabilidade da amostra e idade da mulher.

Portanto, o objetivo deste estudo foi avaliar a eficiência da revisão rápida de $100 \%$ na detecção de resultados falsos-negativos e verificar se esses resultados variam com a adequabilidade da amostra e com a idade da mulher.

\section{Métodos}

Esse estudo foi realizado no Laboratório Rômulo Rocha da Faculdade de Farmácia da Universidade Federal de Goiás (UFG), que analisa, em média, 1.200 esfregaços por mês, os quais são escrutinados e revisados por profissionais especialistas em citopatologia, com experiência que varia de um a 15 anos. No período de março a setembro de 2005, foram analisados 6.008 esfregaços coletados pela técnica convencional para rastreamento de câncer do colo do útero. Desse total, 368 $(6,1 \%)$ esfregaços foram classificados como positivos e $110(1,8 \%)$ com adequabilidade da amostra considerada insatisfatória para análise pelo escrutínio de rotina. O restante dos esfregaços, totalizando 5.530 classificados como negativos foram submetidos à revisão rápida de $100 \%$, utilizando a objetiva de $10 \mathrm{X}$, no tempo médio de um minuto utilizando a técnica Turret ${ }^{11}$, que consiste em reescrutinar toda a lâmina, em uma velocidade regular, percorrendo campos amplos no sentido - vertical - horizontal - vertical - horizontal.

Para avaliar a eficiência do método da revisão rápida de $100 \%$ para detecção de resultados falsos-negativos, 
esse método foi comparado com os métodos de revisão com base em critérios clínicos e revisão aleatória de $10 \%$. Dos 5.530 esfregaços negativos que foram submetidos à revisão rápida de $100 \%$, foram selecionados aqueles que tinham informações com base em critérios clínicos e, em seguida, selecionados aleatoriamente $10 \%$ do total e submetidos aos respectivos métodos de revisões, conforme fluxograma (Figura 1).

$\mathrm{Na}$ revisão rápida de $100 \%$, os esfregaços foram classificados como negativos, insatisfatórios ou suspeitos. $\mathrm{Na}$ revisão com base em critérios clínicos e revisão aleatória de $10 \%$, os esfregaços foram analisados utilizando o tempo similar ao escrutínio de rotina (em média cinco minutos) e classificados de acordo com a alteração encontrada. Os esfregaços classificados como insatisfatórios, suspeitos e alterados foram submetidos à revisão detalhada por dois citologistas. Os resultados concordantes foram considerados como diagnóstico final, enquanto que os resultados divergentes foram analisados por um terceiro citologista, sendo o diagnóstico final definido em reunião de consenso com microscópio multicabeças.

Todas as revisões foram realizadas sem conhecimento dos resultados citopatológicos atribuídos pelos outros citologistas, à exceção da reunião de consenso. A revisão com base em critérios clínicos, revisão aleatória de $10 \%$, adequabilidade da amostra e o diagnóstico final foram classificados de acordo com o Sistema de Bethesda $2001^{17}$.

Participaram desse estudo seis citologistas. Destes, dois eram responsáveis pelo escrutínio de rotina e os outros quatro se alternavam nas revisões rápida de $100 \%$, com base em critérios clínicos, aleatória de $10 \%$ e detalhada para a definição do diagnóstico final.

As proporções entre as taxas de resultados falsosnegativos em função da adequabilidade da amostra dos esfregaços citopatológicos foram comparadas pelo teste do $X^{2}$ e teste de Cochran-Armitage. Foram consideradas significantes as diferenças em que a probabilidadade de rejeição da hipótese de nulidade foi menor que $5 \%$ $(\mathrm{p}<0,05)$. Este estudo foi aprovado pelo Comitê de Ética em Pesquisa da UFG.

\section{Resultados}

Do total de 5.530 esfregaços classificados inicialmente como negativos submetidos à revisão rápida de 100\%, 141 foram classificados como suspeitos, dos quais $84(59,6 \%)$ tiveram algum resultado positivo confirmado. Os diagnósticos mais freqüientes e que, portanto, apresentaram as maiores taxas de resultados falsos-negativos foram células escamosas atípicas de significado indeterminado e lesão intra-epitelial escamosa de baixo grau. Dentre os diagnós-

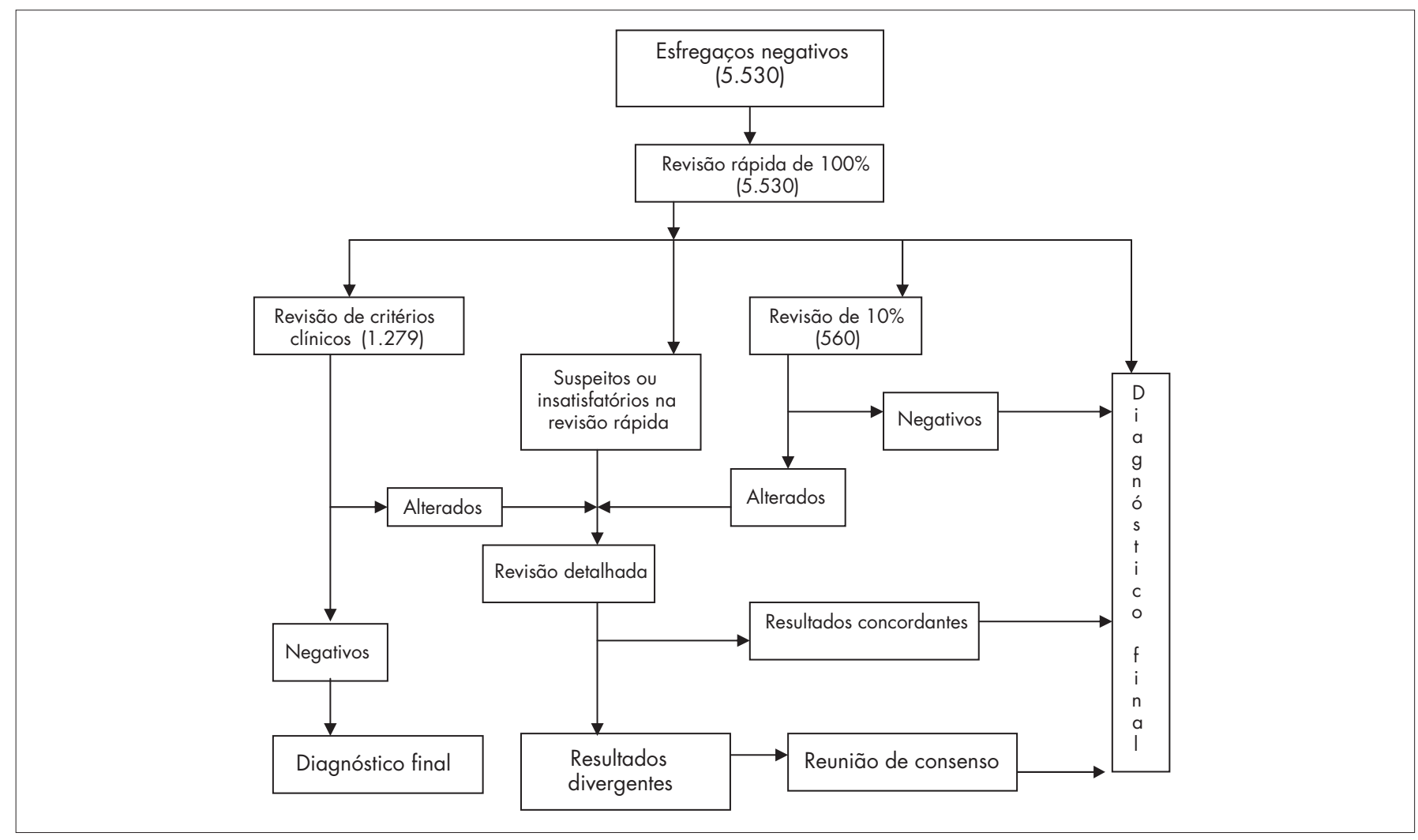

Figura 1 - Fluxograma: métodos de revisão do controle interno da qualidade dos esfregaços cervicais. 
ticos citológicos mais relevantes, cinco $(3,5 \%)$ esfregaços foram classificados como células escamosas atípicas, não podendo excluir lesão de alto grau; seis $(4,3 \%)$ como lesão intra-epitelial de alto grau; e três $(2,1 \%)$ células glandulares atípicas. A revisão rápida de $100 \%$ classificou 19 esfregaços como insatisfatórios, dos quais 16 foram confirmados pelo diagnóstico final (Tabela l).

Os esfregaços falsos-negativos identificados pelos métodos de revisão rápida de $100 \%$, revisão com base em critérios clínicos e revisão aleatória de $10 \%$ foram 84, 19 e seis esfregaços, respectivamente (Tabela 2).

Dos 84 esfregaços suspeitos pelo método de revisão rápida de $100 \%$ e confirmados como alterados pelo diagnóstico final, o escrutínio de rotina classificou 62 como satisfatórios e 22 satisfatórios, porém apresentando alguma limitação para a análise. A freqüência de resultados falsosnegativos dos esfregaços considerados com adequabilidade da amostra satisfatória foi aproximadamente o dobro da freqüência obtida para os esfregaços com alguma limitação, e essa diferença foi significante (Tabela 3).

Os resultados falsos-negativos identificados pela revisão rápida de $100 \%$ não variaram com a idade da mulher $(\mathrm{p}=0,20)$, conforme Tabela 4 .

\section{Discussão}

A freqüência de resultados falsos-negativos identificados pelo método de revisão rápida de $100 \%$ foi de $1,5 \%$, que correspondeu a um acréscimo de quase um quarto de resultados alterados, enquanto que o método de revisão aleatória de $10 \%$ e o método de revisão com base em critérios clínicos detectou 0,1

Tabela 1 - Frequiência de diagnósticos citopatológicos detectados pelo método de revisão rápida de $100 \%$ em esfregaços considerados como negativos no escrutínio de rotina.

\begin{tabular}{|c|c|c|c|c|c|c|c|}
\hline \multirow{3}{*}{$\begin{array}{l}\text { Diagnóstico } \\
\text { final }\end{array}$} & \multicolumn{6}{|c|}{ Revisão rápida } & \multirow{3}{*}{$\begin{array}{c}\text { Frequiência de } \\
\text { esfregaços } \\
\text { FN no } \\
\text { escrutínio de } \\
\text { rotina } \\
\% \\
\end{array}$} \\
\hline & \multicolumn{2}{|c|}{ Suspeito } & \multicolumn{2}{|c|}{ Insutisfatório } & \multicolumn{2}{|c|}{ Total } & \\
\hline & $\mathrm{n}$ & $\%$ & n & $\%$ & $n$ & $\%$ & \\
\hline Negativo & 57 & 40,4 & 3 & 15,8 & 60 & 37,5 & - \\
\hline ASC-US & 36 & 25,5 & $\cdot$ & & 36 & 22,5 & 0,65 \\
\hline ASC-H & 5 & 3,5 & - & & 5 & 3,1 & 0,09 \\
\hline LSIL & 34 & 24,1 & - & & 34 & 21,3 & 0,62 \\
\hline HSIL & 6 & 4,3 & - & & 6 & 3,8 & 0,11 \\
\hline AGC & 3 & 2,1 & - & & 3 & 1,9 & 0,05 \\
\hline INS & 0 & - & 16 & 84,2 & 16 & 10,0 & - \\
\hline Total & 141 & 100 & 19 & 100 & 160 & 100 & 1,52 \\
\hline
\end{tabular}

ASC-US=células escamosas atípicas de significado indeterminado; ASC-H=células escamosas atípicas, não é possível excluir uma lesão intra-epitelial de alto grau; LSIL=lesão intra-epitelial de baixo grau; HSIL=lesão intra-epitelial de alto grau; $\mathrm{AGC}=$ células glandulares atípicas; INS=insatisfatório; $\mathrm{FN}=$ falso-negativo. Foram analisados 5.530 esfregaços pela revisão rápida, dos quais $5.446(98,5 \%)$ foram considerados negativos. A freqüência de resultados falsos-negativos foi calculada tendo como denominador o total de esfregaços analisados. e $0,3 \%$ de lesões, respectivamente. Esses resultados foram consistentes com os resultados de diversos estudos que usaram diferentes desenhos para avaliar o desempenho da revisão rápida. Todavia, todos concluíram que o método é uma alternativa eficiente para reduzir o número de esfregaços falsos-negativos do exame citopatológico ${ }^{8,9,11,12,15}$.

Tabela 2 - Total de esfregacos com resultados falsos-negativos identificados pela revisão rápida de $100 \%$, revisão de critérios clínicos e revisão de $10 \%$.

\begin{tabular}{|c|c|c|c|}
\hline \multirow{2}{*}{$\begin{array}{l}\text { Categoria } \\
\text { diagnóstica }\end{array}$} & \multicolumn{3}{|c|}{ Revisc̃o } \\
\hline & rápida & $\begin{array}{l}\text { de critérios } \\
\text { clínicos }\end{array}$ & de $10 \%$ \\
\hline ASC-US & 36 & 9 & 4 \\
\hline ASC-H & 5 & 1 & 1 \\
\hline LSIL & 34 & 8 & . \\
\hline HSIL & 6 & 1 & 1 \\
\hline AGC & 3 & . & - \\
\hline Total & 84 & 19 & 6 \\
\hline
\end{tabular}

ASC-US=células escamosas atípicas de significado indeterminado; ASC $\mathrm{H}=$ células escamosas atípicas, não é possível excluir uma lesão intra-epitelial de alto grau; LSIL=lesão intra-epitelial de baixo grav; HSIL=lesão intra-epitelial de alto grav; $A G C=$ células glandulares atípicas.

Tabela 3 - Frequiência de esfregaços com resultados falsos-negativos identificados pela revisão rápida de $100 \%$ de acordo com a adequabilidade da amostra.

\begin{tabular}{|c|c|c|c|c|}
\hline \multirow[b]{2}{*}{$\begin{array}{l}\text { Adequabilidade } \\
\text { da amostra }\end{array}$} & \multicolumn{4}{|c|}{ Escrutínio de rotina } \\
\hline & $\begin{array}{l}\text { Resultado } \\
\text { falso } \\
\text { negativo }\end{array}$ & $\begin{array}{c}\text { Resultado } \\
\text { negativo }\end{array}$ & $\begin{array}{l}\text { Esfregaços } \\
\text { analisados }\end{array}$ & $\begin{array}{c}\text { Frequiência de } \\
\text { esfregacos } \\
\text { falsos- } \\
\text { negativos }\end{array}$ \\
\hline $\begin{array}{l}\text { Qualidade } \\
\text { satisfatória }\end{array}$ & 62 & 3.310 & 3.372 & $1,84 \%$ \\
\hline $\begin{array}{l}\text { Qualidade } \\
\text { satisfatória, mas } \\
\text { limitada }\end{array}$ & 22 & 2.117 & 2.139 & $1,03 \%$ \\
\hline Total & 84 & 5.427 & 5.511 & \\
\hline
\end{tabular}

Os 19 esfregaços classificados como insatisfatórios pela revisão rápida foram excluídos da análise.

$x^{2}=5,72 ; p=0,02$.

Tabela 4 - Freqüência de esfregaços falsos-negativos identificados pela revisão rápida de $100 \%$ de acordo com a idade da mulher.

\begin{tabular}{l|ccc}
\hline \multirow{2}{*}{ Idade } & \multicolumn{3}{c}{ Escrutínio de rotina } \\
\cline { 2 - 4 } & $\begin{array}{c}\text { Resultado } \\
\text { negativo }\end{array}$ & $\begin{array}{c}\text { Resultado falso- } \\
\text { negativo }\end{array}$ & Total \\
\hline$\leq 20$ anos & 600 & 12 & 612 \\
21 a 30 anos & 1.654 & 30 & 1.684 \\
31 a 40 anos & 1.354 & 19 & 1.373 \\
41 a 50 anos & 955 & 9 & 964 \\
51 a 60 anns & 572 & 11 & 583 \\
$\geq 61$ anos & 292 & 3 & 295 \\
Total & 5.427 & 84 & 5.511 \\
\hline
\end{tabular}

Os 19 esfregaços classificados como insatisfatórios pela revisão rápida foram excluídos da análise.

Teste de Cochran-Armitage=-1,27; $p=0,20$. 
O método de revisão rápida detectou mais esfregaços falsos-negativos, possivelmente porque o objetivo não foi fornecer um resultado preciso, mas separar esfregaços possivelmente negativos daqueles com suspeita de lesão intra-epitelial. Uma conseqüência esperada foi classificar um número maior de esfregaços suspeitos, que, posteriormente, ao serem revisados detalhadamente foram classificados como negativos ${ }^{18}$. Nesse estudo, observou-se que aproximadamente para cada dois esfregaços suspeitos um foi confirmado como alterado pelo diagnóstico final.

Os diagnósticos falsos-negativos mais freqüentes foram ASC-US e LSIL; este foi um resultado esperado, porque ASC-US e LSIL são diagnósticos limítrofes com a normalidade, e há pelo menos duas razões para esse resultado. A primeira, é que esses diagnósticos são mais freqüentes no escrutínio citopatológico. A segunda pode ser atribuída ao fato de que são alterações celulares mais difíceis de serem identificadas qualitativa e quantitativamente, resultando em baixa concordância interobservadores ${ }^{19}$.

Os resultados deste estudo sugerem que o método de revisão rápida de $100 \%$, ao revisar a totalidade dos esfregaços, aumentou a chance de se identificarem os falsos-negativos, apresentando, então, boa eficiência. Com os outros dois métodos de revisão, obteve-se menor freqüência de resultados falsos-negativos e isso pode ser explicado, tendo em vista que na revisão aleatória de $10 \%$ o falso-negativo pode estar dentre os $90 \%$ não revisados. O método de revisão com base em critérios clínicos, apesar de ter detectado mais falsos-negativos do que a revisão aleatória de $10 \%$, também não revisa a totalidade dos esfregaços negativos. A limitação mais importante do método é que nem sempre as informações clínicas são anotadas ou fidedignas.

Ainda de acordo com os resultados deste estudo, a taxa de resultados falsos-negativos detectados pela revisão rápida foi aproximadamente três vezes maior quando a adequabilidade da amostra foi satisfatória para análise, enquanto que os resultados falsos-negativos de amostras satisfatórias, porém apresentando alguma limitação para a análise, tiveram maior freqüência em esfregaços com ausência de células endocervicais, seguidos de esfregaços obscurecidos por material purulento e espesso.

Os esfregaços com adequabilidade da amostra com alguma limitação para análise podem estar associados a resultados falsos-negativos. A presença de sangue e de processo inflamatório não tem sido sistematicamente associada com maior freqüência de resultados falsosnegativos ${ }^{17,20}$. Um estudo recente, no entanto, mostrou que são fatores que se associaram de maneira indepen- dente com maior risco para resultados falsos-negativos ${ }^{6}$. É possível que esse aparente conflito ocorra devido a diferenças no critério de avaliação da quantidade de sangue e intensidade do processo inflamatório, pois esses fatores dificultam a análise citopatológica ${ }^{17,20}$.

$\mathrm{O}$ método de coleta em meio líquido tem sido apontado como alternativa para superar esta dificuldade de análise do esfregaço preparado pela técnica convencional e assim melhorar a adequabilidade da amostra, diminuir o número de esfregaços insatisfatórios e aumentar a percentagem de diagnóstico de lesões de alto $\operatorname{grau}^{21,22}$, apesar da falta de consenso ${ }^{23}$. As diferentes técnicas de citologia em meio líquido descreveram com detalhes os procedimentos de coleta, normalmente seguidos pelos coletadores ${ }^{24,25}$. A experiência tem mostrado que os profissionais não coletam material para o exame citopatológico convencional com o mesmo rigor e cuidado que o fazem para o método em meio líqui$\mathrm{do}^{23}$. Cabe, então, destacar que a educação permanente para os profissionais responsáveis pela coleta do exame citopatológico é um complemento importante para melhorar a qualidade dos esfregaços.

Este estudo mostrou também que a freqüência dos esfregaços falsos-negativos identificados pela revisão rápida não variou com a idade da mulher, ainda que os resultados falsos-negativos mais freqüentes foram ASC-US e LSIL e em mulheres mais jovens. Por outro lado, a prevalência de lesões mais graves, como a lesão intra-epitelial de alto grau está presente em mulheres com mais de 40 anos e diante das dificuldades relatadas em relação aos esfregaços atróficos, esperava-se encontrar mais resultados falsos-negativos em esfregaços de mulheres mais velhas.

Enfim, os resultados deste estudo mostraram que a revisão rápida de $100 \%$ é uma alternativa eficiente como método de controle interno da qualidade para reduzir as taxas de falsos-negativos do exame citopatológico, e apresentou melhor desempenho quando a amostra foi classificada como satisfatória para análise; todavia, não variou em relação à idade da mulher. Para esfregaços que apresentam alguma limitação para a análise, uma sistemática mais minuciosa de controle de qualidade deveria ser testada, para tentar reduzir ainda mais as taxas de resultados falsos-negativos.

\section{Agradecimentos}

Aos profissionais do Laboratório Rômulo Rocha da Faculdade de Farmácia da UFG e, em especial, a Gislaine Aparecida Fonsechi-Carvasan, da UNICAMP, pela valiosa colaboração na análise dos dados deste estudo. 


\section{Referências}

1. Miller AB, Nazeer S, Fonn S, Brandup-Lukanow A, Rehman R, Cronje $\mathrm{H}$, et al. Report on consensus conference on cervical cancer screening and management. Int J Cancer. 2000;86(3):440-7.

2. Cantor SB, Atkinson EN, Cardenas-Turanzas M, Benedet JL, Follen $M$, MacAulay $C$. Natural history of cervical intraepithelial neoplasia: a meta-analysis. Acta Cytol. 2005;49(4):405-15.

3. Nomenclatura Brasileira para Laudos Cervicais e Condutas Preconizadas recomendações para profissionais de saúde. Rev Bras Ginecol Obstet. 2006;28(8):486-504.

4. Ministério da Saúde. Prevenção do câncer do colo do útero. Manual técnico para laboratórios. Brasília (DF); 2002.

5. Ministério da saúde. Agência Nacional de Vigilância Sanitária. Resolução da Diretoria Colegiada. RDC n 302. Dispõe sobre regulamento técnico para funcionamento de laboratórios clínicos. Brasília (DF); 2005.

6. Franco R, Amaral RG, Montemor EBL, Montis DM, Morais SS, Zeferino LC. Fatores associados a resultados falsos-negativos de exames citopatológicos do colo uterino. Rev Bras Ginecol Obstet. 2006;28(8):479-85.

7. Diehl AR, Prolla JC. Rapid rescreening of cervical smears for internal quality control. Acta Cytol. 1998;42(4):949-53.

8. Amaral RG, Zeferino LC, Hardy E, Westin MC, Martinez EZ, Montenor EB. Quality assurance in cervical smears: $100 \%$ rapid rescreening vs. 10\% random rescreening. Acta Cytol. 2005;49(3):244-8.

9. Michelow P, McKee G, Hlongwane F. Rapid rescreening of cervical smears as a quality control method in a high-risk population. Cytopathology. 2006;17(3): 110-5.

10. Centers for Disease Control. Regulations for implementing the Clinical Laboratory Improvement Amendments of 1988: a summary. MMWR. 1992;41 (RR-2):1-17.

11. Dudding N, Hewer EM, Lancucki L, Rice S. Rapid screening: a comparative study. Cytopathology. 2001;12(4):235-48.

12. Ferraz MG, Dall'Agnol M, Di Loreto C, Pirani WM, Utagawa ML, Pereira SM, et al. $100 \%$ rapid rescreening for quality assurance in a quality control program in a public health cytologic laboratory. Acta Cytol. 2005;49(6):639-43

13. Pajtler M, Audy-Jurkovic S, Skopljanac-Macina L, Antulov J, Barisic A, Milicic-Juhas V. Rapid cervicovaginal smear screening: method of quality control and assessing individual cytotechnologist performance. Cyłopathology. 2006;17(3):121-6.

14. Gupta S, Sodhani P, Singh V, Pant JN, Chachra KL, Bhatt NC, et al. Rapid rescreening of cervical smears cervical by cytopathologists: experience at a $\mathrm{WHO}$ collaborating center for research in cytology Indian J Pathol Microbiol. 2004;47(1):8-10.

15. Manrique EJ, Amaral RG, Souza NL, Tavares SB, Albuquerque ZB, Zeferino $L C$. Evaluation of $100 \%$ rapid rescreening of negative cervical smears as a quality assurance measure. Cytopathology. 2006; 17(3): 1 16-20.

16. Amaral RG, Santos SHR, Catharino JMR, Silva LCB, Westin MCA, Cotta $A C$, et al. Revisão rápida de esfregaços cervicais como método de garantia interna da qualidade. J Bras Patol Med Lab. 2003;39(2):151-5.

17. Solomon D, Nayar R. Sistema Bethesda para citopatologia cervicovaginal. 2a ed. Rio de Janeiro: Revinter; 2005.

18. Lemay C, Meisels A. $100 \%$ rapid (parcial) rescreening for quality assurance. Acta Cytol. 1999;43(1):86-8.

19. Brooke D, Dudding N, Sutton J. Rapid (partial) prescreening of cervical smears: the quality control method of choice? Cytopathology. 2002;13(4):191-9.

20. Phadnis SV, Doshi JS, Ogunnaike OO, Padwick M, Sanusi FA. Inadequate cervical smear: what do we do? Acta Obstet Gynecol Scand. 2005;84(5):486-8.

21. Fremont-Smith M, Marino J, Griffin B, Spencer L, Bolick D. Comparison of the SurePath liquid-based Papanicolaou smear with the conventional Papanicolaou smear in a multisite direct-to-vial study. Cancer. 2004;102(5):269-79.

22. Karnon J, Peters J, Platt J, Chilcott J, McGoogan E, Brewer N. Liquid-based cytology in cervical screening: an updated rapid and systematic review and economic analysis. Health Technol Assess. 2004;8(20):iii, 1-78.

23. Davey E, Barratt A, Irwig L, Chan SF, Macaskill P, Mannes $P$, et al. Effect of study design and quality on unsatisfactory rates, cytology classifications and accuracy in liquid-based versus conventional cervical cytology: a systematic review. Lancet. 2006;367(9505): 122-32.

24. Longatto Filho A, Pereira SM, Di Loreto C, Utagawa ML, Makabe $S$, Maeda MY, et al. DCS liquid-based system is more effective than conventional smears to diagnosis of cervical lesions: Study in high-risk population with biopsy-based confirmation. Gynecol Oncol. 2005;97(2):497-500.

25. Utagawa ML, Pereira SMM, Longatto Filho A, Martins CR, Aguiar LS, Pittoli JE, et al. Citologia de base líquida associada à captura de híbridos para DNA-HPV pode otimizar a qualidade diagnóstica do método de Papanicolaou? Rev Inst Adolfo Lutz. 2004;63(5): 100-3. 